
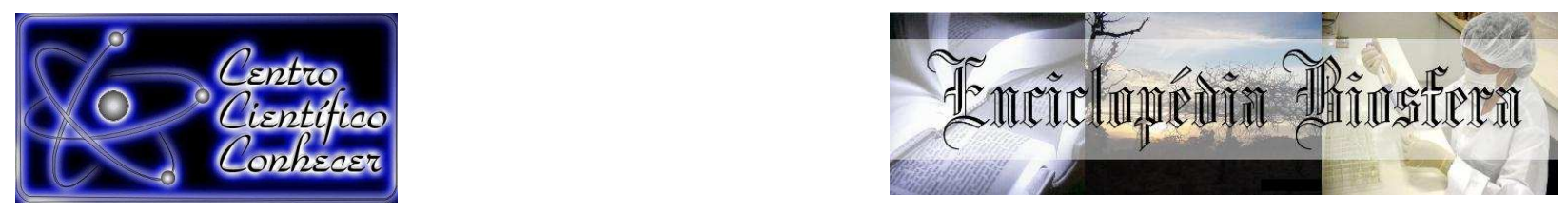

\title{
DESEMPENHO REPRODUTIVO DE FÊMEAS SUÍNAS PRIMÍPARAS SUBMETIDAS OU NÃO À INTERVENÇÃO MANUAL AO PARTO NO SUDOESTE GOIANO
}

Giancarlo Costi ${ }^{1}$, Antonio Sergio Ferraudo ${ }^{2}$
1 Doutorando do Programa de Pós-graduação em Medicina Veterinária da
Universidade Estadual Paulista - UNESP - Jaboticabal
(costi.giancarlo@gmail.com) Jaboticabal - Brasil
Estadual Paulista - UNESP - Jaboticabal
Professor Doutor do Departamento de Ciências Exatas da Universidade
Recebido em: 08/04/2016 - Aprovado em: 30/05/2016 - Publicado em: 20/06/2016
DOI: 10.18677/Enciclopedia_Biosfera_2016_040

\section{RESUMO}

O presente trabalho foi realizado em uma unidade produtora de leitões no sudoeste goiano e buscou caracterizar o desempenho reprodutivo de fêmeas suínas primíparas submetidas ou não à intervenção manual no parto realizada por palpação vaginal. Foram coletadas informações do parto de 1.023 fêmeas suínas de parição 1. As fêmeas foram divididas em grupo Controle $(n=945)$ e Intervenção $(n=78)$. 0 percentual de fêmeas submetidas à intervenção manual no parto foi de $7,6 \%$. Entre as categorias de tamanho de leitegada não houve diferença no percentual de fêmeas com palpação genital durante o parto. $O 4^{\circ}$ trimestre foi o período que houve menos intervenções, enquanto o $2^{\circ}$ trimestre o que teve o maior percentual de intervenções. As primíparas com intervenção no parto apresentaram maior freqüência de fêmeas com pelo menos um natimorto, menor número de leitões nascidos vivos, maior número de leitões natimortos e maior número de leitões mumificados.

PALAVRAS-CHAVE: Desempenho reprodutivo, intervenção manual ao parto, suínos.

\section{REPRODUCTIVE PERFORMANCE OF PRIMIPAROUS SOWS SUBMITTED OR NOT TO MANUAL INTERVENTION AT PARTURITION IN THE SOUTH-WEST AT GOIÁS}

\begin{abstract}
This study was performed in a production of piglets unit in southwest of Goias state and sought to characterize the reproductive performance of primiparous sows submitted or not to manual intervention at the labor parturition realized by vaginal palpation. Farrowing information was collected from 1.023 parity 1 sows. Females were divided into control group $(n=945)$ and intervention $(n=78)$. The percentage of females submitted to manual intervention at farrow was $7.6 \%$. Among the categories of litter size, there was no difference in the percentage of females with genital
\end{abstract}


palpation during the study. The 4th quarter was the period there were fewer interventions, while the 2 nd quarter which had the highest percentage of intervention. Primiparous with intervention at farrowing had higher frequency of females with at least one stillbirth, less piglets born alive, higher number of stillborn and mummified piglets.

KEYWORDS: manual intervention at farrowing, reproductive performance, pigs.

\section{INTRODUÇÃO}

$\mathrm{Na}$ suinocultura tecnificada onde as criações são confinadas e criadas de forma intensiva, a eficiência da matriz pode ser avaliada através de índices como quilogramas de leitão/desmamado/porca, o qual é dependente, dentre outros, do número de leitões nascidos vivos por fêmea (MUIRHEAD \& ALEXANDER, 1997).

Além de se ter um cuidado com a matriz por todo o período de gestação, preocupações em relação ao parto são absolutamente importantes na suinocultura com o objetivo de obter o maior número de leitões nascidos vivos em cada leitegada e manter a saúde reprodutiva da fêmea a fim de dar continuidade à produção de leitões, diminuindo assim o custo de produção e aumentando a lucratividade (KNOX, 2005).

A atenção dada pela pesquisa sobre a assistência ao parto não é muito grande. Isto faz com que os conhecimentos desenvolvidos nas décadas de 60 a 80, sejam em parte utilizados para orientar o manejo da fêmea parturiente, porém é de se supor que o desencadeamento do parto possa ter sofrido algumas mudanças que exigem ações mais acuradas em termos de assistência comparadas aquelas matrizes criadas nas décadas anteriores (WENTZ et al., 2009).

Devido aos investimentos de empresas de genéticas em seus produtos, notaram-se melhoras significativas em relação ao desempenho zootécnico dos suínos nas últimas décadas, porém alguns manejos para as fêmeas gestantes e parturientes, ainda são deficientes, podendo prejudicar o real potencial da matriz (MAGNABOSCO et al., 2011).

Para que seja atingida a produtividade satisfatória em uma unidade de suínos torna-se essencial que todas as atividades desenvolvidas na granja estejam bem sincronizadas, atendendo às técnicas de manejo recomendadas com adequado treinamento de funcionários, e que o sistema de coleta de dados, fundamental para a formação exata dos índices de produção, seja correto (SCHNEIDER et al., 2004).

A taxa de natimortalidade em granjas tecnificadas fica em torno de 7 a $9 \%$, podendo ser classificada em natimortos pré-parto, intraparto e pós-nascimento (BORTOLOZZO et al., 2003). Estudos baseados em necropsias afirmam que aproximadamente $10 \%$ dos natimortos morrem no período pré-parto, $75 \%$ no intraparto e 15\% logo após o nascimento (WENTZ et al., 2006). Natimortos pósnascimento compreendem leitões que nasceram vivos e morreram logo após, possivelmente por falta de assistência na fase neonatal, ou por lesões cerebrais devido a anóxia ou hipóxia durante o parto (KNOX, 2005). Dessa forma, nota-se a importância do alto percentual de natimortos intraparto, que constitui o maior grupo de perdas (MELAGGl et al., 2007).

As distocias em suínos ocorrem em menos de 3\% dos partos, não sendo comuns quando comparadas às outras espécies (SMITH,1997). Os fatores envolvidos na ocorrência de partos distócicos são inércia uterina, mau posicionamento do leitão no canal do parto, presença de mais de um feto no canal, deslocamento uterino e fetos muito grandes em relação ao canal do parto (BRITT et 
al., 1999). A distocia aumenta a incidência de leitões natimortos, principalmente por prolongar a duração do parto (JACKSON, 1975). Com a correta assistência ao parto, é possível reduzir a ocorrência de natimortalidade, aumentando o número de leitões nascidos vivos (KNOX, 2005). Para melhor atender a parturiente, é necessário compreender os mecanismos que envolvem o desencadeamento do parto e quais os eventos que ocorrem durante o mesmo (BERNARDI, 2007; MELAGGI et al., 2009), e interferindo quando for absolutamente necessário de acordo com uma recomendação para cada evento, no sentido de prevenir futuros problemas produtivos e reprodutivos (WENTZ et al., 2009).

Quando o intervalo entre os nascimentos exceder de 20-30 minutos, recomenda-se realizar a palpação genital para evitar a exaustão da fêmea (KNOX, 2005; MEREDITH, 1995). Caso necessário, uma intervenção precoce é importante devido à grande perda de leitões por leitões natimortos intraparto, chegando até $72,2 \%$ do total de natimortos (SCHNEIDER et al., 2001). Aproximadamente $93 \%$ dos natimortos intraparto morrem devido à hipóxia causada pela ruptura prematura do cordão umbilical (KNOX, 2005). De acordo com BRITT et al. (1999), $20 \%$ de leitões natimortos são associados a casos de distocias. Desta forma, a intervenção manual precoce é necessária, principalmente, quando o intervalo entre os nascimentos é superior a 20 a 30 minutos (MELAGGI et al., 2007).

A intervenção inclui exame manual da vagina, cerviz ou até onde se consegue palpar, removendo obstáculos de obstrução ou fetos mal apresentados (BRITT, et al., 1999). Para estes autores, a aplicação de antimicrobiano nem sempre é efetiva na prevenção de infecções uterinas. Segundo KLOPFENSTEIN et al., (1999), a palpação genital nem sempre é uma prática segura, aumentando o risco de problemas lactacionais precoces, além de secreções vulvares e endometrites. As secreções vulvares não purulentas pós-parto são consideradas fisiológicas, pois resultam da eliminação dos remanescentes da placenta e restos celulares, visando promover a limpeza uterina (MacLACHLAN \& FOLEY, 1996; BRITT et al., 1999).

Há pouca informação disponível até o momento sobre as conseqüências envolvidas na intervenção do trato genital. $\mathrm{O}$ trabalho desenvolvido visou avaliar o efeito da palpação genital em partos de primíparas sobre o desempenho reprodutivo de acordo com o tamanho da leitegada e trimestre do ano.

\section{MATERIAL E MÉTODOS}

O trabalho foi realizado em uma granja de produção de leitões localizada no sudoeste goiano, no período de outubro de 2014 a novembro de 2015. Foram colhidas as informações das fichas de 1.023 fêmeas de ordem de parto 1. Para avaliar o efeito da intervenção pela palpação genital, as matrizes foram divididas em grupo controle $(n=945)$ e palpação $(n=78)$. Os dados coletados referentes ao parto foram data do parto, número de leitões nascidos totais (NT), número de leitões nascidos vivos (NV), natimortos (NM), mumificados (MM) e ocorrência ou não de palpação genital.

As fêmeas foram categorizadas conforme tamanho da leitegada em leitões nascidos totais $<12,12-15$ e $>15$. Os partos foram agrupados em quatro períodos (trimestres) do ano, denominados em quente e ameno. O período considerado quente compreende as estações primavera/ verão ou $4^{\circ}$ e $1^{\circ}$ trimestres, respectivamente, enquanto o período ameno, as estações outono/ inverno ou $2^{\circ}$ e $3^{\circ}$ trimestres, respectivamente. 
Os critérios para a palpação genital foram determinados pela distocia, ou seja, conforme a dificuldade da fêmea ao trabalho de parto. Os funcionários da granja eram devidamente treinados para o atendimento ao parto e realização da palpação vaginal. Dessa forma, primíparas com partos prolongados, aumento nos intervalos entre nascidos ou contrações sem o nascimento de leitões receberam assistência obstétrica com palpação genital.

Os percentuais de fêmeas com intervenção ao parto de acordo com o tamanho da leitegada e período do ano, além da freqüência de fêmeas com pelo menos um natimorto foram comparados pelo teste do qui-quadrado através do software SPSS Statistics. Para análise do número de leitões nascidos totais e nascidos vivos as médias foram comparadas pelo teste t através do SPSS Statistics. Os natimortos e mumificados foram analisados pelo teste de Wilcoxon pelo programa Biostat 5.0.

\section{RESULTADOS E DISCUSSÃO}

O percentual de primíparas submetidas à intervenção manual no parto foi de $7,6 \%$, próximo dos $10 \%$ que SOBESTIANSKY et al., (2001) consideram que a taxa de palpação genital deve ficar, a fim de evitar intervenções desnecessárias, mas diferente dos resultados encontrados por MELLAGI et al., (2009) onde foi encontrado um percentual de $20,6 \%$ e de $22,8 \%$ observado em estudo realizado por BORGES et al., (2005), ambos efetuados em granjas brasileiras.

Não houve diferença no percentual de fêmeas com intervenção manual entre as classes de tamanho da leitegada (Tabela 1)

TABELA 1. Percentual de primíparas submetidas ou não à intervenção manual ao parto de acordo com as categorias de tamanho de leitegada e período do ano.

\begin{tabular}{ccc}
\hline Categorias & Controle & Intervenção \\
\cline { 2 - 3 } & $\mathbf{9 2 , 4 \% ( 9 4 5 )}$ & $\mathbf{7 , 6 \% ( 7 8 )}$ \\
\hline Tamanho da leitegada & & \\
\hline$<12$ & $19,8 \%(187)$ & $21,8 \%(17)$ \\
$12-15$ & $52,1 \%(492)$ & $48,7 \%(38)$ \\
$>15$ & $28,1 \%(266)$ & $29,5 \%(23)$ \\
Período do ano & & \\
\hline $1^{\circ}$ trimestre & $21,5 \%(203)$ & $20,5 \%(16)$ \\
$2^{\circ}$ trimestre & $25,1 \%(237)$ & $34,6 \%(27)^{\star}$ \\
$4^{\circ}$ trimestre & $26,2 \%(248)$ & $28,2 \%(22)$ \\
& $27,2 \%(257)$ & $16,7 \%(13)^{\star}$ \\
\hline
\end{tabular}

${ }^{*}$ Significativo a $5 \%$ de probabilidade pelo teste binomial

Houve diferença entre os grupos $(P<0,05)$ controle e intervenção no segundo e quarto trimestres do ano. No segundo trimestre a freqüência de intervenções foi maior, enquanto que no quarto trimestre foi a menor. Em trabalho realizado por MELAGGI et al. (2007) foi encontrada diferença $(P<0,05)$ no percentual de intervenções entre todas as estações do ano. Em estudo realizado por PRIVADO FILHO, (2010) no sudoeste goiano, nos dois períodos quentes as médias das taxas de natimortos $(4,9 \%$ e $5,4 \%)$ foram maiores em relação aos períodos amenos $(4,2$ e $4,4 \%)$. 
Entre as classes de tamanho de leitegada, não foi observada diferença no percentual de matrizes com palpação genital durante o parto $(P>0,05)$. BORGES et al. (2005) encontraram índices de intervenção diferentes de acordo com a classe de tamanho da leitegada. As fêmeas com menos de 10 leitões tiveram $32 \%$ de intervenção manual, superior aos $24 \%$ e $18 \%$ para as fêmeas com 10-12 e >12 leitões, respectivamente. Segundo os autores, em leitegadas com poucos leitões, a intervenção manual teria sido conduzida para certificar que não havia mais leitões no canal do parto.

As características das fêmeas, no momento do parto, submetidas ou não à intervenção manual, podem ser vistas na Tabela 2. As primíparas com intervenção no parto apresentaram maior freqüência de fêmeas com pelo menos um natimorto no parto $(\mathrm{P}<0,001)$ quando comparadas às fêmeas do grupo controle, maior número de leitões natimortos $(P<0,001)$ e maior número de leitões mumificados $(P<0,05)$ por leitegada. Não foi observada diferença entre as variáveis para leitões nascidos totais e vivos $(P>0,05)$. Em estudo realizado por MELAGGl et al. (2009) também foi encontrado maior número de leitões natimortos por leitegada e maior freqüência de fêmeas com pelo menos um natimorto no parto $(P<0,001)$, porém foi observada diferença entre os grupos para leitões nascidos totais e mumificados, menor média de leitões nascidos vivos.

TABELA 2. Características reprodutivas ao parto das primíparas submetidas ou não à intervenção manual ao parto.

\begin{tabular}{lccc}
\hline \multicolumn{1}{c}{ Variáveis } & Controle $(\mathbf{n = 9 4 5 )}$ & Intervenção $(\mathbf{n = 7 8 )}$ & $\mathbf{P}$ \\
\hline Fêmeas com natimortos & $35,6 \%(336)$ & $56,4 \%(44)$ & $<0,001$ \\
Leitões nascidos totais & $13,7 \pm 3,1$ & $13,9 \pm 3,3$ & 0,594 \\
Leitões nascidos vivos & $12,8 \pm 3,0$ & $12,2 \pm 3,4$ & 0,098 \\
Natimortos & $0,5 \pm 0,8$ & $1,1 \pm 1,4$ & $<0,001$ \\
Mumificados & $0,3 \pm 1,0$ & $0,6 \pm 1,0$ & $<0,047$ \\
\hline
\end{tabular}

\section{CONCLUSÕES}

A intervenção manual ao parto em primíparas é realizada com maior frequência no $2^{\circ}$ trimestre e com menor frequência no $4^{\circ}$ trimestre. O tamanho da leitegada (12-15) influenciou no percentual de intervenção. Nas fêmeas submetidas à intervenção manual, a freqüência de com natimortos é maior. O parto é mais prolongado e o número de natimortos é maior.

\section{REFERÊNCIAS}

BERNARDI, M. L. Fisiologia do parto em suínos. Acta Scientiae Veterinariae [online], v.35, p.139-147, ISSN 1679-9216, 2007.

BORGES, V.F., BERNARDI, M. L.; BORTOLOZZO, F. P.; WENTZ, I. Risk factors for stillbirth and foetal mummification in four Brazilian swine herds. Preventive Veterinary Medicine, v.70, p.165-176, 2005.

BORTOLOZZO, F. P. B.; WENTZ, I.; BORGES, V. F.; SCHNEIDER, L. G.; BERCELOS, D. E. S. N. Natimortalidade na suinocultura tecnificada: a importância de um diagnóstico correto. Suinocultura em foco, n.9, p. 3-5, 2003. 
BRITT, J.H.; ALMOND, G. W.; FLOWERS, W. L. Diseases of the Reproductive System. In: STRAW, B. E. et al. Diseases of Swine. $8^{a} \mathrm{ed}$. London: lowa State University Press, p.883-911, 1999.

JACKSON. P. G. G. The incidence of stillbirth in cases of dystocia in sows. Veterinary Record, v. 97, p. 411-412. 1975.

KNOX, R. V. Improving Farrowing Management. Ohio Pork Information Center. Disponível em: http://www.livestocktrail.illinois.edu/swinerepronet/publications/extension/FarrowJan2 01.pdf. Acessado em 27 outubro 2012.

KLOPFENSTEIN, C.; FARMER, C.; MARTINEAU, G.P. Diseases of the mammary glands and lactation problems. In: LEMAN, A. D.; STRAW, B. E.; MENGELING, W. L.; D'ALLAIRE, S.; TAYLOR, D. J. Diseases of swine. 8. ed. London: lowa State University Press, p. 833-860, 1999.

MacLACHLAN, N. J.; FOLEY, G. L. The female reproductive tract. In: SIMS, L. D., GLASTONBURY, J. R. W. Pathology of pig. A diagnostic guide. Australia: The Pig research and Development Corporation, p. 385-400, 1996.

MAGNABOSCO, D., RIBEIRO, R. R., BIERHALS, T., BORTOLOZZO, F. P., WENTZ, I Fatores envolvidos na preparação das matrizes para o parto. Disponível em: http://www.porkworld.com.br/artigos/post/fatores-envolvidos-napreparacao-das-matrizes-para-o-parto. Acessado em 13 outubro 2012.

MELLAGI, A. P. G; FURTADO, C. S. D; CYPRIANO, C. R; HEIM, G; BERNARDI, M L; BORTOLOZZO, F. P.; WENTZ, I. Procedimentos e consequências das intervenções manuais ao parto em suínos. Acta Scientiae Veterinariae [online], v.35, p.149-156, ISSN 1679-9216, 2007.

MELLAGI, A. P. G; HEIM, G; BERNARDI, M L; BORTOLOZZO, F. P.; WENTZ, I. Caracterização e desempenho reprodutivo de fêmeas suínas submetidas à intervenção obstétrica manual. Ciência Rural, v.39, n.5, p.1478-1484, 2009.

MEREDITH, M. J. Pig breeding and infertility. In: Animal Breeding and Infertility. Blackwell Science, 1995. p.278-353, 1995.

MUIRHEAD, M.R. \& ALEXANDER, T. J. L. Managing pig health and the treatment of disease. A reference for the farm. Sheffield: $5 \mathrm{M}$ Enterprises, p. 133-186, 227282.1997.

PRIVADO FILHO, J. R. Aspectos reprodutivos de fêmeas suínas primíparas e secundíparas em Rio Verde - GO. 2010. 52 f. Tese (Doutorado em Medicina Veterinária) - Faculdade de Ciências Agrárias e Veterinárias, Universidade Estadual Paulista "Júlio de Mesquita Filho", Jaboticabal, 2010.

SCHNEIDER, L. G.; COSTI, G.; BORTOLOZZO, F. P. Avaliação da mumificação fetal e natimortalidade de acordo com o tamanho da leitegada e ordem de parto. In: 
CONGRESSO BRASILEIRO DE VETERINÁRIOS ESPECIALISTAS EM SUÍNOS, 10., 2001, Porto Alegre. Anais. Porto Alegre: Associação Brasileira de Veterinários Especialistas em Suínos (ABRAVES), v. 2, p. 199-200, 2001.

SCHNEIDER, L. G.; BORTOLOZZO, F. P.; WENTZ, I.; BORCHARDT NETO, G. Erros de anotações na elaboração de índices de produção em granjas industriais de suínos no Sul do Brasil. Arq. Bras. Med. Vet. Zootec. [online]. vol.56, n.1, pp. 8185. ISSN 0102-0935, 2004.

SMITH C.A. Normal and abnornal parturition in swine. In: Youngquist R.S. (Ed.). Current therapy in large animal theriogenology. Philadelphia: W.B. Saunders Company, p. 719-726. 1997.

SOBESTIANSKY, J.; BARCELOS, D. E. S. N.; MORENO, A. M.; CARVALHO, L. F. O. S. Visita ao sistema de produção. In: SOBESTIANSKY, J; BARCELLOS. Clínica veterinária em sistemas intensivos de produção de suínos e relatos de casos clínicos. Goiânia: Gráfica Art 3. p. 28-58, 2001.

WENTZ, I.; CYPRIANO, C. R.; VARGAS, A. J.; BERNARDI, M. L; BORTOLOZZO, F. $P$. Fatores de risco para leitões natimortos e mumificados. In: CONGRESSO LATINO- AMERICANO DE SUINOCULTURA, 3, 2006. Foz do Iguaçu. Anais... Foz do Iguaçu. p 271-287, 2006.

WENTZ, I.; BIERHALS, T.; MELLAGI, A. P. G.; BORTOLOZZO, F. P.; A importância do atendimento ao parto na melhoria da produtividade em suínos. Acta Scientiae Veterinariae. v.37, p.35-47, 2009. 